\title{
Determinação de idade cronológica de Calliphoridae (Diptera) através da deposição pós-emergencial de quitina.
}

\author{
Emygdio L. A. Monteiro Filho ${ }^{1}$
}

\begin{abstract}
A method for chronological age determination based on mesothoracic post-phragma post-emergence deposition of chitin is used for Chrysomya putoria and Phaenicia cuprina (Diptera, Calliphoridae). The age of these species were obtained by the maximum period of deposition which were of 11-12 days.
\end{abstract}

A importância da determinação da idade cronológica dos animais torna-se evidente quando da necessidade destes dados em estudos ecológicos e epidemiológicos. Métodos para a determinação da idade cronológica foram reunidos por SOUTHWOOD (1978), mas em 1962 contudo, DETINOVA já havia reunido vários métodos para a determinação da idade fisiológica de insetos de interesse médico, baseados principalmente em alterações observadas durante o desenvolvimento ovariano que, entretanto, não podem ser aplicados a machos e a espécimes fixados ou conservados.

NEVILLE em 1963, trabalhando com ortópteros, mostrou a ocorrência de deposições pós-emergenciais diárias de quitina e verificou haver relação entre as camadas de deposição e o tempo de vida dos individuos. SCHLEIN \& GRATZ (1972) relatam a utilização de deposições pós-emergenciais endocuticulares de quitina na região dos pós-fragmas mesotorácicos de dípteros Nematocera, sendo este método mais tarde também aplicado a espécimes da familia Sarcophagidae (SCHLEIN, 1972, 1975) e à Glossina (SCHLEIN, 1979). Deposição pós-emergencial de quitina no apódema do ducto ejaculatório foi pela primeira vez utilizada por DREW (1969) em Dacus tryoni e foi posteriormente aplicado a outros Tephritidae (KAMALI \& SCHULZ, 1974) tendo porém, a desvantagem de não poder ser aplicado às fêmeas. Mais recentemente, ELLISON \& HAMPTON (1982) utilizando-se de outra estrutura torácica, a terceira furca, obtiveram bons resultados com uma espécie pertericente à familia Calliphoridae.

Neste trabalho, procurou-se testar a utilização da deposição pós-emergencial de quitina nos pós-fragmas mesotorácicos para a datação de idade cronológica de outros dïpteros Ciclorrafos utilizando para este estudo duas espécies de moscas pertencentes à familia Calliphoridae .

${ }^{1}$ Departamento de Zoologia do Instituto de Biologia, UNICAMP, CP. 6109 - 13.081 Campinas, SP. Bolsista do CNPq. 


\section{MATERIAL E MÉTODOS}

Serviram como objeto deste estudo, moscas pertencentes às espécies Chrysomya putoria e Phaenicia cuprina, sendo o experimento efetuado em duas fases. A primeira realizada com espécimes criados em condições laboratoriais, como controle, e a outra que correspondeu às análises do material coletado no campo ou proveniente de coleções cientifíicas.

Animais de ambas as espécies foram mantidos à temperatura de $25^{\circ} \mathrm{C} \pm$ $1^{\circ} \mathrm{C}$, em gaiolas com armação de ferro $(32 \mathrm{~cm} \times 32 \mathrm{~cm} \mathrm{x} 48 \mathrm{~cm}$ ) revestidas com tela de nailom; água e alimento foram fornecidos "ad libitum"; a dieta era constituida por levedura de cerveja em pó, leite integral em pó e açúcar $(1: 1: 1)$. Diariamente eram retiradas dez moscas de ambos os sexos, fixadas e conservadas em álcool a $70 \%$ para preparações microscópicas e tomada de medidas da endocutícula. A coleta de moscas no campo foi feita com uma armadilha semelhante à usada por FERREIRA (1978); como iscas utilizou-se carcaças de animais, como por exemplo: camundongo, sapo e peixe. Os individuos coletados eram fixados e conservados em álcool a $70 \%$ ou em congelador $\left(-10^{\circ} \mathrm{C}\right)$.

$\mathrm{Na}$ preparação do material, os exemplares eram imersos em solução de $\mathrm{KOH}$ a $10 \%$ a frio durante 48 horas ou então, a quente por 10 minutos, facilitando a retirada da musculatura e iniciando a clarificação. Depois de lavado em álcool a 70\%, o abdomem era separado do tórax, sendo este seccionado entre o segundo e o terceiro par de patas com o auxilio de uma lâmina metálica fina. Após, era feita a retirada dos pós-fragmas mesotorácicos que eram colocados em líquido de Hoyer entre lâmina e lamínula. O material assim preparado foi medido com o auxilio de ocular milimetrada em um microscópio, com um aumento de 80 vezes.

Posteriormente foram feitas regressões das medidas obtidas dos pósfragmas mesotorácicos ao longo dos dias, seguindo as seguintes equações: para $P$. cuprina, $\mathrm{y}=0.021534+0.0162063$. $\mathrm{x}$ e para C. putoria, $\mathrm{y}=0.0557728+$ $0.0143409 . \mathrm{x}$.

\section{RESULTADOS E DISCUSSÃO}

A utilização de padrões de deposição diária da endocutǐcula pós-emergencial de quitina mostrou ser aplicável a alguns insetos (NEVILLE, 1963; SCHLEIN \& GRATZ, 1972; DINGLE et al., 1969); contudo, a espessura desta deposição varia dependendo da luminosidade (DINGLE et al., 1969; SCHLEIN, 1975, 1979; TYNDALE-BISCOE \& KITCHING, 1974), assim como do fornecimento de alimentação proteica; entretanto, foi demonstrado por TYNDALEBISCOE \& KITCHING (1974) não ser esta influência significativa, pois é muito pequena ou até mesmo inexiste em alguns dias.

A deposição pós-emergencial de quitina dos pós-fragmas mesotorácicos

que já podia ser determinada em dìpteros Nematocera, Sarcophagidae, Glossinidae e Calliphoridae, pode também ser determinada para C. putoria e $P$. cuprina. Em espécimes criados em laboratório, observou-se que houve um crescimento, altamente significativo, das deposições pós-emergenciais de quitina ( $P$. cuprina, $\mathrm{r}^{2}=0.832,(\mathrm{P}<0.01)$ e $C$. putoria, $\mathrm{r}^{2}=0.939,(\mathrm{P}<0.01)$ 


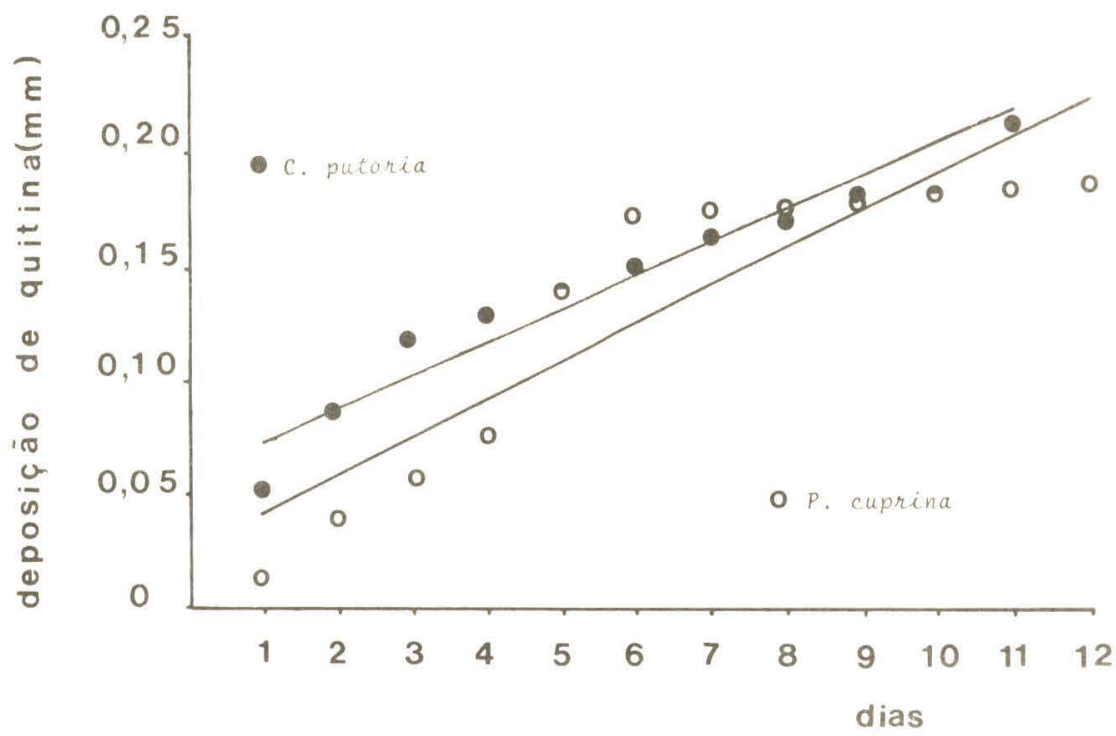

FIG. 1 - Medidas obtidas a partir da deposição pós-emergencial de quitina nos pós-fragmas mesotorácicos ao longo dos dias. A reta de regressão superior corresponde à $C$, putoria, enquanto a de baix o, à $P$. cuprina .

para as duas espécies (Fig. 1 ), estando esta deposição correlacionada com o periodo que variou de 11 a 12 dias, dependendo da espécie. Dados semelhantes foram obtidos por TYNDALE-BISCOE \& KITCHING (1974) utilizando individuos de P. cuprina dorsalis, da Austrália. Estes autores verificaram que a deposição aumenta até nove ou dez dias, dependendo de fatores como temperatura e luminosidade, tendo este crescimento atingido aproximadamente $0.18 \mathrm{~mm}$ à temperatura de $25^{\circ} \mathrm{C} \pm 1^{\circ} \mathrm{C}$.

Como nos resultados existentes para $P$. cuprina dorsalis (TYNDALEBISCOE \& KITCHING, 1974) os dados de laboratório e de campo são semelhantes, será considerado que a mesma semelhança deva ocorrer para as espécies agora estudadas. Desta forma, verificou-se que para C. putoria, cerca de $73,69 \%$ dos indivíduos coletados no campo, encontravam-se com mais de dez dias de vida e apenas $26.31 \%$ estavam na faixa de idade que vai de um a dez dias, enquanto para $P$. cuprina os resultados foram bem diferentes, pois $48.15 \%$ dos indivíduos encontravam-se com mais de dez dias de vida e $51.85 \%$ variavam entre um e dez dias, sendo que destes, $64.29 \%$ estavam na faixa correspondente ao quinto dia de vida.

Este método, contudo, mostrou não ser aplicável além destes periodos, pois as espessuras das deposições começam a variar muito de indivíduo para indivǐduo, tornando insegura a avaliação dos resultados. 
Pelo visto acima, é possĩvel supor que parte da população estudada de C. putoria, eram adultos com mais de dez dias e que a maior parte da população de $P$. cuprina estava com menos de dez dias de vida.

\section{AGRADECIMENTOS}

Agradeço ao Dr. Ângelo Pires do Prado, do Departamento de Parasitologia da UNICAMP pela orientação e amizade sempre demonstrada durante a realização deste trabalho. A Karin D. Kempers pela leitura do manuscrito e apoio.

\section{BIBLIOGRAFIA}

DETINOVA, T. S. 1962. Methods of determining the physiological age of female Anopheles. Age grouping methods in Diptera of medical importance. World Health Organ. Monograph Ser. 47:69-77.

DINGLE, H.; R. L. CALDWELL \& J.B. HASKELL. 1969. Temperature and circadian control of cuticle grow th in the bug, Oncopeltus fasciatus. J. Insect Physiol. 15:373-378.

DREW, T. A. I. 1969. Morphology of the reproductive system of Strumera tryoni (Froggatt) (Diptera: Trypetidae) with a method os fistinguishing sexually mature adult males. J. Aust. Entomol. Soc. 8:21-32.

ELLISON, J.R. \& E. N.HAMPTON. 1982. Age determination using the apodeme structure in adult screwworm flies (Cochliomyia hominivorax). J. Insect Physiol. 28(9):731-736.

FERREIRA, M. J. M. 1978. Sinantropia de díptero muscóideos de Curitiba, Paraná. I: Calliphoridae. Revta bras. Biol. 38(2):445 454.

KAMALI, K. \& J. T. SCHULZ. 1974. Biology and ecology of Gymnocarena diddusa (Diptera: Tephritidae) on sunflower in Dakota. Ann. Ent. Soc. Amer. 67(4): 695-699.

NEVILLE, A. C. 1963. Daily grow th layers in locust rubber like cuticle influenced by an ex ternal rhy thm. J. Insect Physiol. 9:177-186.

SCHLEIN, Y. 1972. Factors that influence the post-emergence growth in Sacophaga falculata. J. Insect Physiol. 18:199-209.

SCHLEIN, Y. 1975. Effects of U. V. light and temperature on the melanization and the formation of daily grow th layers of Sarcophaga faculata.J.Insect Physiol.21:1859-1863.

SCHLEIN, Y. 1979. Age grading of tse tse flies by the cuticular growth layers in the thoracic phragma, Ann. Trop. Med, Parasitol. 73(3) 297-298.

SCHLEIN, Y. \& N. G. GRATZ. 1972. Age determination of some flies and mosquitoes by daily grow th layers of skeletal apodemes. Bull. World Health Organ. 47:71-76.

SOUTHWOOD, T. R. E. 1978. Ecological Methods. Chapman and Hall, London. 524 pp.

TYNDALE-BISCOE, M.\& R. L. KITCHING. 1974. Cuticular bands age criteria in the sheep blowfly Lucilia cuprina (Wied) (Diptera: Calliphoridae). Bull. Entomol. Res. 64: 161-174. 